msh-mss Mathématiques et sciences humaines

181 | Printemps 2008

Varia

\title{
Éléments pour une ethnomathématique de l'awélé
}

Aspects of the ethnomathematics of the game awele

Marc Chemillier

\section{OpenEdition}

Journals

Édition électronique

URL : http://journals.openedition.org/msh/8883

DOI : $10.4000 / \mathrm{msh} .8883$

ISSN : 1950-6821

\section{Éditeur}

Centre d'analyse et de mathématique sociales de l'EHESS

\section{Édition imprimée}

Date de publication : 20 mars 2008

Pagination : 5-34

ISSN : 0987-6936

\section{Référence électronique}

Marc Chemillier, «Éléments pour une ethnomathématique de l'awélé », Mathématiques et sciences humaines [En ligne], 181 | Printemps 2008, mis en ligne le 27 mars 2008, consulté le 23 juillet 2020 URL : http://journals.openedition.org/msh/8883 ; DOI : https://doi.org/10.4000/msh.8883 


\title{
ÉLÉMENTS POUR UNE ETHNOMATHÉMATIQUE DE L’AWÉLÉ
}

\author{
Marc CHEMILLIER $^{1}$
}

\begin{abstract}
RÉSUMÉ - L'ethnomathématique étudie certaines activités de sociétés de tradition orale, qui portent sur des nombres, des formes géométriques, ou des arrangements d'éléments, et s'apparentent à des constructions mathématiques. Le plus souvent, ces activités ne font l'objet d'aucune verbalisation, et il est difficile de savoir ce que "pensent» ceux qui les pratiquent. Les jeux de stratégie, comme le jeu africain awélé, sont une exception, car les joueurs peuvent expliquer leurs stratégies. Le but de cet article est de comparer certaines propriétés mathématiques de l'awélé et le discours autochtone des experts, pour évaluer la distance qui sépare ces deux points de vue.

MOTS-CLÉS - Awélé, Cognition, Ethnomathématique, Jeux, Stratégies

SUMMARY - Aspects of the ethnomathematics of the game awele

Ethnomathematics is a new domain focusing on activities of traditional societies based on mathematical concepts such as numbers, forms, arrangements. Generally speaking, these activities are not associated with spoken descriptions from people doing them. It is thus difficult to analyse the way they conceive the mathematical notions underlying them. A game such as the awele played in Africa is an exception, since players can explain their strategies. The purpose of this article is to compare some mathematical properties of awele, and some explanations given by players of this game, in order to evaluate the distance between these two points of view.
\end{abstract}

KEY-WORDS - Awele, Cognition, Ethnomathematics, Game, Strategies

Certaines activités pratiquées dans les sociétés de tradition orale reposent sur des idées proches des objets élémentaires utilisés dans les mathématiques occidentales (nombres, formes géométriques, arrangements d'éléments), et consistent à effectuer sur ces objets des manipulations systématiques et assez complexes. L'étude de ces activités et la mise en évidence de leur contenu proprement mathématique ont donné naissance il $\mathrm{y}$ a quelques décennies à un nouveau champ de l'histoire et de la didactique des mathématiques appelé ethnomathématique [Ascher, 1986].

Le terme «ethnomathématique» peut surprendre. Les mathématiques ne consistent-elles pas à définir des concepts universaux dans une langue universelle ? Comment dans ces conditions peut-on lier les termes «mathématique » et « ethno » comme si l'on voulait désigner de cette manière certaines formes de raisonnement rationnel restant étrangères à la discipline ? En réalité, pour universelles qu'elles soient, les mathématiques n’en ont pas moins une histoire associée à une géographie. Cette

1 Groupe de Recherche en Informatique, Image, Automatique et Instrumentation de Caen (GREYC), (UMR 6072), Université de Caen, 14032 Caen, et Centre d'Analyse et de Mathématique Sociales (CAMS), UMR 8557 EHESS-CNRS, 54 boulevard Raspail 75270 Paris cedex 06, chemilli@free.fr 
science apparaît comme le fruit des traditions de différentes régions du monde grecque, arabe, chinoise ou indienne - qui ont apporté au fil du temps leur pierre à l'édifice. L'histoire des mathématiques se donne pour but d'étudier ces traditions écrites savantes. L'ethnomathématique, elle, étend le champ d'investigation à des traditions non écrites, et en ce sens, elle en est un prolongement.

L'une des difficultés qui se posent dans les études au sein de sociétés de tradition orale est de relier les descriptions des propriétés observées (qui sont décrites dans le langage des mathématiques occidentales contemporaines) avec les processus mentaux effectivement mis en œuvre au sein de la société étudiée. Le plus souvent, les activités donnant lieu à ces élaborations mathématiques ne font l'objet d'aucune verbalisation. Il n'existe pas, ou peu, de discours autochtone à leur sujet. Ainsi, dans le contexte des sociétés de tradition orale, les situations permettant d'étudier les modalités du raisonnement hypothético-déductif sont rares, car de nombreux savoirs ne sont pas associés à des verbalisations. Le cas des jeux de stratégie constitue une exception. Le principe même de la succession des coups entre les deux joueurs fournit un cadre propice à la déduction et à l'anticipation qui sont les outils de leurs stratégies.

L'awélé est un jeu de semailles répandu sur tout le continent africain, dont il existe de nombreuses variantes. Il présente un cas intéressant pour étudier la verbalisation et les raisonnements associés à des savoirs mathématiques dans les sociétés de tradition orale. En effet, on peut comparer les propriétés mathématiques du jeu et de ses différentes configurations d'une part, et le discours autochtone des experts de ce jeu tel qu'on peut réellement l'observer sur le terrain d'autre part, et de cette façon, évaluer la distance qui sépare ces deux points de vue.

Plusieurs études ont été consacrées aux propriétés formelles de l'awélé. Par exemple, l'ethnomathématicien Ron Eglash a montré que certaines successions de coups faisaient apparaître des configurations stables (qui se reproduisent indéfiniment), d'autres alternant périodiquement. Il est possible que les joueurs experts africains aient plus ou moins conscience de ces propriétés et qu'ils s'en servent dans leur stratégie. Mais ce point ne peut être véritablement acquis que si l'on effectue une enquête de terrain pour recueillir leurs raisonnements sur leur pratique. Il se trouve que de telles enquêtes ont commencé à se développer, notamment dans les travaux de psychologie interculturelle menés par Jean Retschitzki.

Notre hypothèse sera la suivante :

(H0) Les raisonnements de mathématiciens occidentaux étudiant certaines configurations de l'awélé sont semblables sur plusieurs points à ceux d'experts africains analysant les stratégies du jeu.

Pour étayer cette hypothèse, nous allons dans un premier temps rappeler les règles $\mathrm{du}$ jeu. Puis nous décrirons quelques résultats obtenus par des chercheurs occidentaux mathématiciens ou informaticiens à propos de certaines configurations de graines de l'awélé. Ensuite, nous présenterons certains raisonnements de joueurs ivoiriens tels qu'ils sont décrits dans les travaux de Jean Retschitzki. Ceux-ci concerneront plus particulièrement les fins de parties, où le nombre de graines est réduit et où il est plus facile de repérer certaines dispositions de graines ayant des propriétés particulières. On verra qu'il existe des similitudes entre ces configurations étudiées par les joueurs et celles analysées par les mathématiciens. Toute l'enjeu de cette approche comparative sera de préciser ce qu'on entend par «semblables» lorsqu'on rapproche ainsi le 
discours des uns et des autres, autrement dit de dégager les traits généraux que ces deux types de raisonnements peuvent avoir en commun ${ }^{2}$.

\section{INTRODUCTION}

\subsection{DÉMONSTRATION ET RAISONNEMENT LOGIQUE}

On sait la place du raisonnement hypothético-déductif dans le développement des mathématiques depuis l'Antiquité. Les Grecs sont responsables de l'introduction en mathématiques d'une approche de type dialectique, qui repose principalement sur la logique déductive, et qui s'intéresse à des questions d'existence [Davis, Hersh, 1985, p. 181]. Un exemple de cette approche est l'affirmation par Pythagore vers 550 av. J.C. selon laquelle il n'existe pas de solution rationnelle à l'équation $x^{2}=2$. Une telle affirmation s'écarte notablement $\mathrm{du}$ point de vue consistant à calculer des approximations successives de la racine carrée de deux. Dans le processus de formalisation des mathématiques qui a dominé le $\mathrm{XX}^{\mathrm{e}}$ siècle, l'approche dialectique est devenue prépondérante. L'une de ses caractéristiques principales est l'usage généralisé de la démonstration logique, au point que l'on considère parfois qu'il n'y a pas de mathématiques hors du schème hypothético-déductif propre à la démonstration.

Mais ce point de vue tend à reléguer au second plan la notion d'algorithme suite d'opérations reproductibles sans ambiguïté dans différents contextes - qui est pourtant plus ancienne et plus universelle. Il existe en effet une autre approche des mathématiques, de type algorithmique, qui suit des méthodes de calcul explicite de solutions ou de leurs approximations. Cette approche est sans doute beaucoup plus répandue à l'échelle de l'humanité. Près de deux mille ans avant J.C., les Babyloniens savaient déjà calculer une dizaine de décimales de la racine carrée de deux. Notons qu'aujourd'hui, le développement des ordinateurs suscite un nouvel essor de l'approche algorithmique.

Il est évident que dans les sociétés de tradition orale, les mathématiques sousjacentes à certaines activités à caractère technique sont plutôt de type algorithmique. Mais cela ne dispense pas de s'interroger sur la manière dont le schème hypothéticodéductif est pratiqué dans ces sociétés à travers des raisonnements verbalisés. Cette question appartient aujourd'hui au champ des sciences cognitives. Plus précisément, elle concerne les recherches interculturelles qui se sont développées en leur sein durant les dernières décennies. De telles recherches ont jusqu'à présent beaucoup porté sur le problème de la déduction syllogistique. L'exemple suivant, qui ressemble à un « dialogue de sourds », met bien en lumière les difficultés qui se posent quand on veut isoler le plan purement logique du discours dans une enquête de terrain [Boyer, 1986] :

«-L'enquêteur :

Tous les Kpelle cultivent le riz.

\footnotetext{
${ }^{2}$ Ce texte reprend sous une forme développée et mise à jour certains éléments d'un exposé présenté aux Rencontres musicales pluridisciplinaires du Grame à Lyon en mars 1996 [Chemillier, 1997]. Une autre version du même texte, moins formalisée, est publiée dans un chapitre du livre Les Mathématiques naturelles [Chemillier, 2007(a)]. Les problèmes abordés ici, concernant le lien entre ethnomathématique et représentations mentales autochtones, prolongent un autre article intitulé « Mathématiques de tradition orale » [Chemillier, 2007(b)], où nous présentons les résultats d'une enquête ethnomathématique de terrain que nous avons menée à Madagascar dans le cadre d'une recherche sur les mathématiques de la divination.
} 
M. Smith ne cultive pas le riz.

Question : M. Smith est-il un Kpelle ?

- L'indigène :

Je ne connais pas M. Smith, je ne l'ai jamais vu ».

L'analyse de ce dialogue classique a conduit à diverses interprétations, mais la thèse de l'incapacité des sujets à maîtriser la déduction syllogistique ne semble plus guère défendue. En revanche, l'importance de la différence culturelle entre l'enquêteur et l'enquêté est mieux prise en compte, et l'on évalue plus justement la distorsion qu'elle introduit dans le déroulement de l'enquête [Scribner, 1977]. Ce qui était parfois perçu comme illogique est aujourd'hui plus prudemment considéré comme incomplet, c'est-à-dire comme faisant référence à des propositions sous-entendues dont l'elision empêche de saisir la logique du discours.

\subsection{L’AWÉLÉ}

\subsubsection{Description du jeu}

Les jeux de stratégie constituent un objet propice à l'étude des mécanismes de raisonnement. En effet, les jeux de ce type se déroulent à l'intérieur d'un univers abstrait de cases et de pions, en marge du monde réel, avec des règles de fonctionnement explicites. Ils incluent dans leur principe même la notion de séquentialité (alternance régulière de coups) qui leur confère une affinité avec le schème linéaire du raisonnement déductif (« si je joue cela, il se passe ceci »).

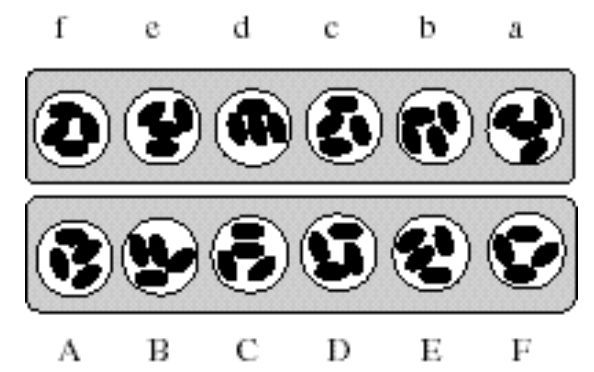

FIGURE 1. Le tablier de l'awélé avec six cases dans chaque camp

L'Afrique est le continent d'un jeu aussi passionnant et raffiné que les échecs ou le go, appelé « jeu des semailles ». Il se présente sous la forme d'un tablier creusé de trous dans lesquels sont distribuées des graines. Il en existe une grande variété, que l'on regroupe en deux types: les jeux de type wari (ou awélé, owari, etc.) dont le tablier comporte deux rangées de cases et qui sont largement répandus en Afrique occidentale ; et les jeux de type solo (ou soro, kisoro, etc.) à trois ou quatre rangées de cases, qui sont pratiqués principalement en pays bantou [Deledicq, Popova, 1977].

Le plus connu des jeux de semailles est l'awélé. C'est un jeu de type wari, comportant deux rangées de six cases contenant initialement quatre graines par case. Un coup consiste à prendre le contenu d'une case de son camp (la rangée placée de son côté) et à tourner dans le sens anti-horaire le long des deux rangées (la sienne et celle de l'adversaire) en distribuant une graine par case. Par convention, on notera par des majuscules les cases de Sud et par des minuscules celles de Nord (de la gauche vers la droite pour chacun des joueurs). Si Nord joue la case « $\mathrm{d}$ », il sème quatre graines dans les cases «e », «f», «A $\mathrm{A}$ et « $\mathrm{B} »($ Figure 2$)$. 


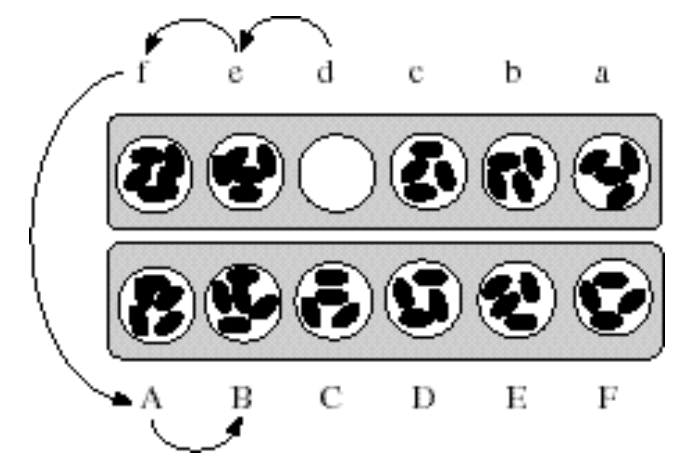

FIGURE 2. En jouant la case « $\mathrm{d} »$, Nord sème quatre graines dans les cases « $\mathrm{e} », \ll \mathrm{f} », \ll \mathrm{A} »$ et $\ll \mathrm{B} »$ en tournant dans le sens anti-horaire

On notera une position du tablier par deux lignes de chiffres indiquant les contenus des deux rangées de cases. La position initiale et celle obtenue après le coup précédent s'écrivent donc :

\section{4 \\ 444444 \\ 550444 \\ 554444 .}

Il y a prise quand un coup se termine dans une case du camp adverse dont le contenu est porté à deux ou trois graines. Dans ce cas, on récolte le contenu de cette case, ainsi que celui de toutes les cases précédentes du camp adverse qui contiennent également deux ou trois graines. Par exemple, considérons la succession théorique de coups suivante « $\mathrm{FaE} »$ avec trait à Sud à partir de la position obtenue après le coup précédent.

\section{5}

554440

051555

665550

052666

665501 .

On voit qu'en jouant « $\mathrm{E} »$ au bout de quatre coups, Sud capture deux graines dans la case $« d » d u$ camp adverse (Figure 3 ).

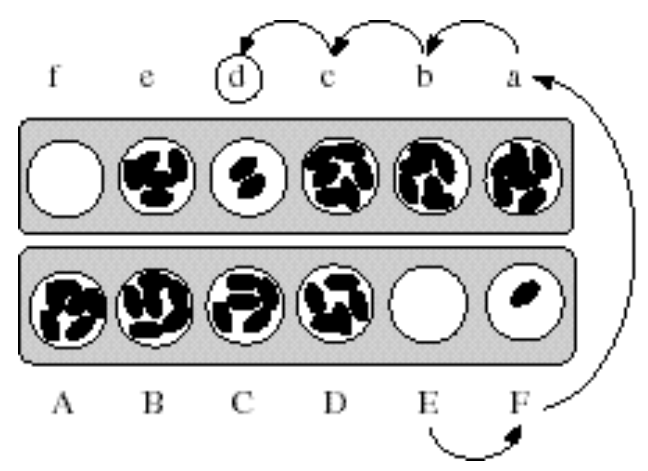

FIGURE 3. En jouant «E $»$, Sud capture deux graines dans la case « $\mathrm{d} »$ du camp adverse 
La partie se termine quand il ne reste plus assez de graines en jeu pour permettre des prises, ou bien quand un joueur n'a plus de graine dans son camp sans que l'adversaire puisse jouer un coup qui lui en redonne. Dans ce cas, l'adversaire prend toutes les graines restantes. On notera que le joueur qui peut redonner des graines à un adversaire qui n'en a plus est obligé de le faire. Le gagnant est celui qui a récolté le plus de graines.

Comme c'est souvent le cas dans les sociétés de tradition orale, l'awélé prend place au sein d'un réseau de correspondances symboliques. Dans ces sociétés, en effet, les domaines d'activité de la pensée ne sont jamais indépendants les uns des autres. Par exemple, chez les Nzakara de République centrafricaine, qui pratiquent une variante du jeu appelée $a-n g u^{n} l a ̀$ (c'est-à-dire " les bois »), de type solo (quatre rangées de huit cases contenant initialement deux graines par case), ces correspondances prennent la forme d'une métaphore de la guerre. Éric de Dampierre écrit à ce sujet : «[Les joueurs] donnent aux mouvements du jeu une interprétation qui invoque, symboliquement et matériellement, la stratégie et la tactique des armées zandé et nzakara » [Dampierre]. Mais l'existence de cette interprétation symbolique n'altère en rien la cohérence et le caractère systématique de la logique sous-jacente du jeu. Or c'est précisément ce plan purement logique que nous nous efforçons de dégager, et sur lequel nous allons placer notre analyse. Il est important de souligner que cet effort de dissociation des plans de la pensée ne va pas sans difficultés. Les indigènes ne pratiquent ni la logique formelle, ni les mathématiques en tant que discipline autonome. Chaque notion véhicule toujours de nombreuses significations, que l'enquêteur doit apprendre à neutraliser s'il veut mettre en évidence les aspects proprement logique ou mathématique attachés à ces notions.

L'un des premiers ouvrages d'ethnographie décrivant les régles de l'awélé est le livre collectif Religion and Art in Ashanti publié en 1927 par l'anthropologue britannique Robert S. Rattray, qui a longtemps travaillé au Ghana. Ce livre contient un chapitre intitulé « Wari » consacré à la description de l'awélé rédigé par G.T. Bennett, à qui Rattray avait appris le jeu ${ }^{3}$. Outre les règles, il présente également quelques raisonnements logiques décrivant les stratégies du jeu, essentiellement dans les fins de partie.

\subsubsection{Définition des mouvements lents}

Dans les fins de partie, Bennett définit ce qu'il appelle un «mouvement lent ». Lorsqu'il ne reste plus beaucoup de graines en jeu, le joueur a intérêt à en garder beaucoup de son côté, et à en laisser le moins possible du côté de l'adversaire. Un mouvement lent consiste alors à répartir les graines de son camp dans le plus de cases possible, et à jouer toujours la case qui a le moins de graines. Il donne l'exemple suivant :

0000000

3000001

représenté Figure 4.

Sud doit donner une graine à Nord, c'est-à-dire jouer « $\mathrm{F} »$, puis Nord joue « $\mathrm{a} »$. Ensuite, Sud joue la case « $\mathrm{A} »$ avec trois graines, et Nord joue « $\mathrm{b} »$ :

0000100

011100 ${ }^{3}$ Le texte de Bennett a été la source de beaucoup d'autres études sur l'awélé et reproduit de nombreuses
fois (par exemple dans [Kovach, 1995]). 


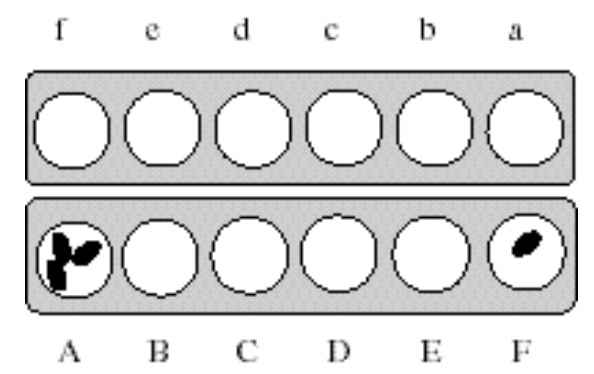

FIGURE 4. Point de départ d'un «mouvement lent » en fin de partie

Dès lors, Sud joue le mouvement lent suivant : « DcCdBeC». Ainsi, il garde toutes ses graines de son côté sans en placer dans «F », ce qui l'obligerait à en donner à Nord.

1000000

000210

Nord est obligé de jouer «f » et n'a plus de graines. Sud n'étant pas en mesure de lui en donner, la partie s'arrête et Sud ramasse les quatre graines. Bennett conclut en disant que «tout autre mouvement rendrait impossible la capture de ces quatre graines ».

\subsubsection{Définition des groupes de marche}

Une autre notion introduite par Bennett, également utile dans les fins de partie, est celle de «groupe de marche ». Il définit ainsi une succession de $n$ cases consécutives dont les nombres de graines sont décroissants de $n$ jusqu'à 1 , c'est-à-dire $n, n-1, \ldots 2,1$. Une telle configuration a la propriété de se déplacer vers la droite sans se modifier quand on joue la case de gauche. Par exemple, dans le groupe de marche 4321 , si le joueur joue la case contenant quatre graines, il les sème dans les cases suivantes, ce qui donne la même configuration 4321 déplacée d'une case vers la droite (Figure 5).

Bennett indique que le groupe de marche 21 , avec deux cases, est utile lorsque l'adversaire n'a pas de graines à gauche de son camp, car en le déplaçant jusqu'au camp adverse, il permet de gagner deux graines. L'exemple qu'il donne met en œuvre plusieurs groupes de marche successifs de ce type :

00000000

0111121

Sud déplace le premier groupe de marche en jouant « $\mathrm{E} »$ :

00000001

0111102

puis la succession « $\mathrm{aF} »$ permet à Sud de prendre deux graines. Ensuite, Sud peut reconstituer un nouveau groupe de marche en jouant « $\mathrm{aDbCcBdCe} »$ :

1000000

000210

Puis Sud déplace le nouveau groupe de marche deux fois jusque dans le camp adverse et prend deux graines comme précédemment « $\mathrm{DfEaF} »$ :

0000001

1000000 


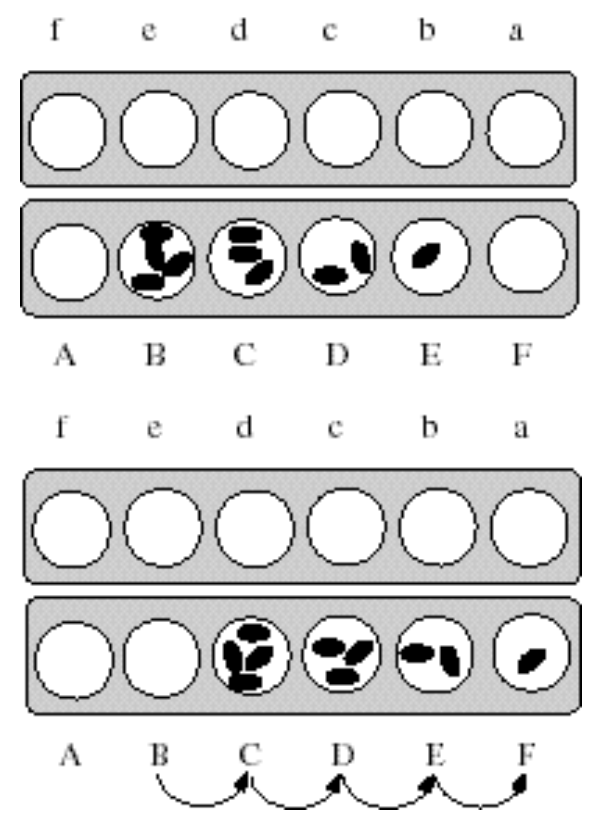

FIGURE 5. Le « groupe de marche » 4321 qui reste identique après semis de sa case de gauche

Nous allons voir que ces notions introduites par Bennett mettent en évidence des propriétés mathématiques intéressantes, qui seront étudiées plus loin. Mais de notre point de vue, qui consiste à relier propriétés mathématiques et modes de pensée locaux, il manque un maillon entre le texte de Bennett et les conceptions des indigènes qui pratiquent ce jeu. En effet, Bennett ne donne pas d'indication sur les conditions dans lesquelles les informations qu'il présente ont été recueillies sur le terrain. Par exemple, on ne dispose d'aucun terme vernaculaire désignant les notions telles que " groupe de marche » ou «mouvement lent». Les exemples analysés ci-dessus sont-ils des extraits de parties observées chez les Ashanti ? Peuvent-ils être considérés comme des « cas d'école » décrits par des experts indigènes ? Dans une certaine mesure, il est possible que Bennett ait imaginé ces situations lui-même en explorant le jeu, comme il le laisse entendre dans son introduction : «Après un peu d'expérience dans la pratique du jeu, on verra que les éléments décrits ici constituent une petite partie des considérations très complexes qui déterminent les meilleurs mouvements »[Bennett, 1927]. Rappelons également que Bennett n'a pas, semble-t-il, effectué d'enquête sur le terrain lui-même, son travail étant fondé sur les données recueillies par Rattray.

\subsection{MATHÉMATISATION DES JEUX ET CONFIGURATIONS REMARQUABLES}

Un jeu peut être représenté par un graphe dont les sommets sont les états possibles (un état est une répartition particulière des pions sur le tablier), et les transitions correspondent aux coups permettant de passer d'un état à un autre. L'étude de tels graphes est l'un des objectifs de la théorie des jeux ${ }^{4}$. Évidemment, pour des jeux comme les échecs ou le go, le nombre de sommets du graphe est considérable. Ce paramètre donne une indication sur la complexité du jeu, car il permet de se faire une idée du

\footnotetext{
${ }^{4}$ La partie de la théorie des jeux concernée par les jeux de pions est le sujet de la thèse de Claude Berge [Berge, 1957]. Une référence plus récente est l'ouvrage de [Conway, 1976]. Pour une étude des jeux envisagée sous un angle plus général, voir les ouvrages classiques [Huizinga, 1938], [Callois, 1967].
} 
nombre de situations qu'il faudrait idéalement envisager pour déterminer une stratégie gagnante ${ }^{5}$.

S'il est difficile de mettre en évidence des propriétés générales du graphe décrivant toutes les situations possibles, il est plus facile d'étudier les propriétés de configurations particulières. Dans les fins de partie, par exemple, le nombre de pièces restantes est souvent réduit, et il est parfois possible, pour certaines configurations remarquables, de mener une analyse plus poussée des choix qui s'offrent aux joueurs. La mathématisation ne porte plus alors sur le graphe du jeu dans son ensemble, mais sur certains sous-graphes dont on met en évidence des propriétés spécifiques. De ce point de vue, l'awélé semble être un champ de recherche particulièrement riche, car ses règles sont simples et ses pièces non différenciées, contrairement à celles des échecs, par exemple, qui obéissent à des règles différentes selon qu'il s'agit des tours, des chevaux, des fous, etc. Cela explique sans doute pourquoi plusieurs mathématiciens ont exploré les propriétés de ce jeu en portant plus particulièrement leur attention sur les configurations de fin de partie. Il se trouve que différentes configurations de ce type, à nombre réduit de graines, ont révélé des propriétés mathématiques remarquables (par exemple les groupes de marche introduits par Bennett). La section qui suit est consacrée à l'exposé de ces travaux réalisés par des mathématiciens occidentaux sous prétexte de l'awélé.

\section{ANALYSE MATHÉMATIQUE DE CONFIGURATIONS DE FIN DE PARTIE}

\subsection{GROUPES DE MARCHE ET CONFIGURATIONS PÉRIODIQUES}

\subsubsection{Déplacement des configurations de l'awélé}

L'ethnomathématicien Ron Eglash a étudié les groupes de marche de l'awélé et plus généralement les configurations du jeu qui sont périodiques [Eglash, 2005] ${ }^{6}$. Cette analyse est le point de départ de développements mathématiques élaborés, dont le lien avec la pratique du jeu pourra sembler ténu. Ce lien repose, en fait, sur le contexte dans lequel Bennett a introduit les «groupes de marche», à savoir pour analyser les stratégies possibles du jeu en fin de partie.

L'analyse d'Eglash suppose que la configuration de graines est placée sur une rangée infinie de cases. Bien entendu, cela ne correspond pas à la réalité du tablier de l'awélé. Mais il est commode de faire ce genre d'hypothèse pour mettre en évidence certaines régularités, afin d'en comprendre les causes et d'en proposer un modèle mathématique. La Figure 6 montre la configuration 342 1. Lors du semis de la case de gauche « $\mathrm{B} »$, le contenu des cases $\langle\mathrm{C} »,\langle\mathrm{D} »$ et $« \mathrm{E} »$ est modifié. On obtient alors la configuration 532 . On notera ce déplacement sous la forme :

$$
3421 \rightarrow 532
$$

Le tablier étant supposé infini, on peut répéter indéfiniment le semis de la case de gauche.

\footnotetext{
${ }^{5}$ La maîtrise du graphe complet de l'awélé à l'aide d'un ordinateur a été réalisée en 2003 par deux chercheurs néerlandais [Romein, Bal, 2003].

${ }^{6}$ Son analyse repose sur la notion d'automate cellulaire (cf. [Wolfram, 1984]).
} 


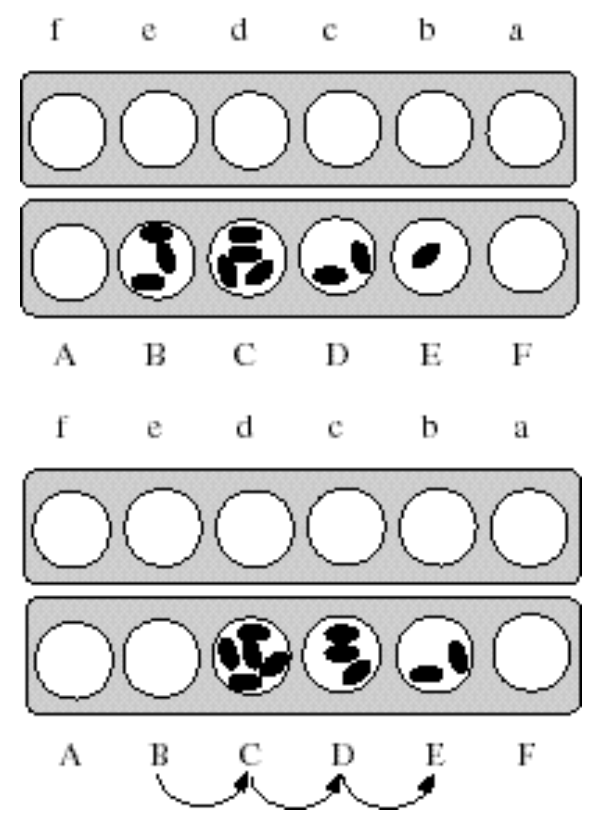

FIGURE 6. La configuration 3421 se transforme en 532 après semis de sa case de gauche

Les groupes de marche de l'awélé sont un exemple de configuration autoreproductrice (le semis de la case de gauche les laisse inchangés, cf. Figure 5). D'autres configurations ont un comportement qui les amène à converger vers un groupe de marche. La configuration 3421 de la Figure 6 est précisément un exemple de ce type cité par Ron Eglash. En semant la case de gauche, on obtient 53 2. Si l'on réitère le semis plusieurs fois (en semant toujours la case de gauche), on constate que la configuration se transforme après plusieurs étapes en 4321 , qui est un groupe de marche. À partir de cet état, le semis ne produit plus aucune modification, car le groupe de marche est un état stable :

$$
\begin{aligned}
& 3421 \rightarrow 532 \rightarrow 43111 \rightarrow 4222 \rightarrow 3331 \rightarrow 442 \rightarrow 5311 \rightarrow \\
& 42211 \rightarrow 3322 \rightarrow 433 \rightarrow 4411 \rightarrow 5221 \rightarrow 33211 \rightarrow 4321
\end{aligned}
$$

La règle de déplacement peut aussi conduire à des comportements périodiques. C'est le cas de la configuration 211 . Lorsqu'on sème plusieurs fois la case de gauche, on obtient d'abord 22 , puis 31 , puis on revient à 211 . Cet exemple est donc de période trois :

$$
211 \rightarrow 22 \rightarrow 31 \rightarrow 211 .
$$

Comme l'ensemble des formes que peut prendre une configuration donnée lors de ses déplacements est fini, on retombe nécessairement à un certain moment sur une forme déjà vue précédemment. Dès lors, les déplacements ultérieurs adopteront un comportement périodique, qui constitue une sorte de cycle limite de la configuration initiale, et apparaît après un premier épisode transitoire. On montre que la période du régime périodique dépend du nombre de graines de la configuration. Les valeurs correspondantes sont regroupées dans le tableau ci-après. Par exemple, la configuration 3421 contient dix graines, le tableau indique que son régime périodique est de période un, c'est-à-dire qu'elle converge nécessairement vers un groupe de marche, ce qui confirme le constat précédent. 


\begin{tabular}{|c|c|c|c|c|c|c|c|c|c|c|c|c|c|}
\hline Nombre de graines & 1 & 2 & 3 & 4 & 5 & 6 & 7 & 8 & 9 & 10 & 11 & 12 & 13 \\
\hline Périodes possibles & 1 & 2 & 1 & 3 & 3 & 1 & 4 & 4,2 & 4 & 1 & 5 & 5 & 5 \\
\hline
\end{tabular}

TABLEAU 1. Périodes des configurations de l'awélé en fonction de leur nombre de graines

L'article de Ron Eglash, paru dans le numéro spécial d'avril 2005 du magazine Pour la science consacré aux «Mathématiques exotiques » qui présentait son analyse des groupes de marche de l'awélé, a eu un effet de catalyseur en donnant naissance à plusieurs études de chercheurs mathématiciens ou informaticiens consacrées aux propriétés des groupes de marche et des configurations périodiques de ce jeu. L'une des belles réussites dans ce domaine est un joli résultat établi par André Bouchet, professeur d'université aujourd'hui à la retraite, spécialiste de combinatoire, qui montre que les configurations périodiques de l'awélé sont exactement les positions appelées «groupes de marche augmentés », qui sont «prises en sandwich » entre deux groupes de marche dont les nombres de cases sont deux entiers consécutifs.

\subsubsection{Définition des groupes de marche augmentés}

André Bouchet définit ce qu'il appelle un «groupe de marche augmenté » [Bouchet, 2005]. Il s'agit d'une configuration obtenue à partir d'un groupe de marche, en ajoutant une graine dans certaines cases, ou éventuellement dans la case à droite de la dernière case du groupe. La Figure 7 montre le groupe de marche augmenté 553211 . Il est obtenu à partir du groupe de marche 54321 disposé dans les cases « $\mathrm{A}$ » à « $\mathrm{E}$ » en ajoutant une graine dans « $\mathrm{B} »$ (qui passe de quatre à cinq graines) et dans « $\mathrm{F} »$ (qui passe de zéro à une graine).

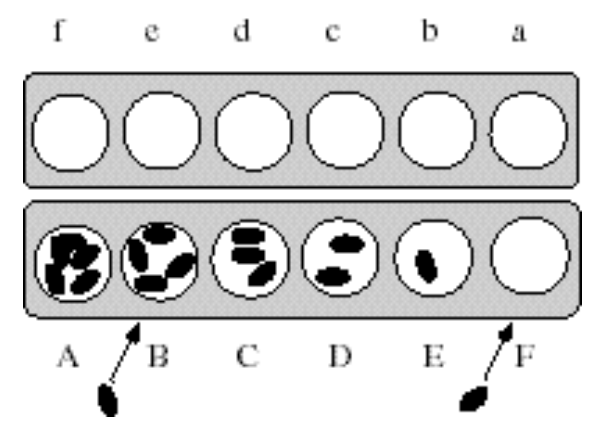

FIGURE 7. Le « groupe de marche augmenté » 553211 obtenu à partir de

54321 en ajoutant une graine dans « $\mathrm{B} »$ et $« \mathrm{~F} »$

Si l'on ajoutait exactement une graine dans toutes les cases indiquées dans la définition ci-dessus (celles du groupe de marche, plus une case supplémentaire à droite de la dernière), on en ajouterait également dans les cases « $\mathrm{A} »,\langle\mathrm{C} »,\langle\mathrm{D} »$ et $\langle\mathrm{E} »$. Mais on obtiendrait alors le groupe de marche 654321 dont le nombre de cases est immédiatement supérieur au précédent. En ce sens, on peut dire qu'un groupe de marche augmenté, au sens de Bouchet, est compris entre deux groupes de marche dont les nombres de cases sont des entiers consécutifs, et qui le prennent «en sandwich ». On notera que les groupes de marche sont des cas particuliers de groupes de marche augmentés. 


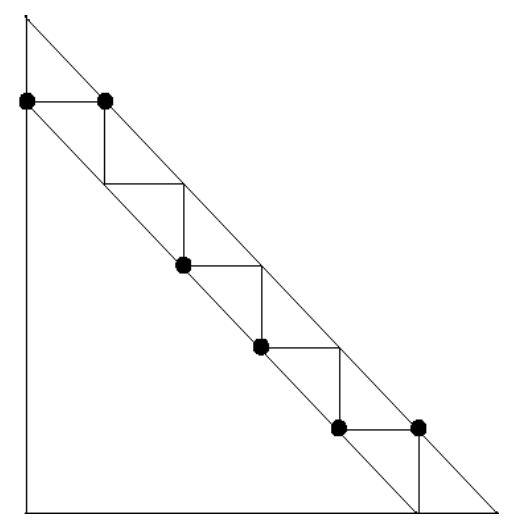

FIGURE 8. Le « groupe de marche augmenté » 553211 est pris en sandwich entre les deux groupes de marche 54321 et 654321 , de cinq et six cases respectivement

\subsection{3. Équivalence entre groupes de marche augmentés et configurations périodiques}

Le résultat de Bouchet établit que les configurations périodiques de l'awélé sont exactement les groupes de marche augmentés. Il est facile de voir qu'une telle configuration est périodique. Par définition des groupes de marche augmentés, elle «contient» un groupe de marche sous-jacent, auquel on a ajouté une graine dans certaines cases. Lorsqu' on joue sa case de gauche, on reconstitue, à une case de distance vers la droite, une nouvelle configuration qui contient, elle aussi, un groupe de marche sous-jacent avec une graine supplémentaire dans certaines cases. Or si l'on considère la séquence de zéro et de un indiquant la présence ou l'absence de cette graine supplémentaire, on observe que, lors de ce déplacement, la séquence subit exactement une permutation circulaire d'un élément, c'est-à-dire que son premier élément est placé à la fin. Par exemple, pour la configuration périodique 553211 (Figure 7), on obtient les déplacements suivants :

$$
\begin{gathered}
553211 \rightarrow 64322 \rightarrow 543311 \rightarrow 54422 \rightarrow \\
55331 \rightarrow 64421 \rightarrow 553211 .
\end{gathered}
$$

Le groupe de marche sous-jacent est 54321 . Pour la séquence de zéro ou un indiquant les graines additionnelles, on obtient les permutations circulaires suivantes :

$$
\begin{gathered}
010001 \rightarrow 100010 \rightarrow 000101 \rightarrow 001010 \rightarrow 010100 \rightarrow \\
101000 \rightarrow 010001 .
\end{gathered}
$$

En conséquence, en réitèrant le processus, tous les éléments de cette séquence vont être déplacés à la fin les uns après les autres, et on reviendra à la séquence initiale.

Il est plus difficile de montrer que toute configuration périodique est nécessairement un groupe de marche augmenté. Pour cette partie du théorème, la preuve originale de Bouchet a été simplifiée, par l'auteur lui-même, ainsi que par un autre chercheur Henning Bruhn, actuellement post-doctorant à l'Université de Hambourg. Ce dernier, dont on reproduit le raisonnement ci-après, a également étendu le résultat [Bruhn, 2005]. 


\subsection{ANALYSE DE LA PREUVE DE BRUHN}

\subsubsection{Groupe de marche minorant et suite des différences}

Nous allons consacrer une large place à la démonstration de Bruhn pour une raison bien précise : nous voulons montrer de cette façon que, même si cette preuve est un raisonnement élaboré, elle ne fait appel à aucun concept mathématique spécialisé. De ce fait, elle constitue un exemple de raisonnement dont l'apparition dans un contexte sans écriture serait théoriquement possible, au moins sous une forme simplifiée. Autrement dit, ce raisonnement ne comporte pas d'éléments qui, sur le plan cognitif, se révèleraient incompatibles avec les conditions de la pure oralité.

L'idée d'Henning Bruhn est de considérer à partir d'une configuration donnée (périodique ou non), le groupe de marche le plus grand possible qui la « minore », c'està-dire tel que toutes ses cases aient moins de graines que les cases correspondantes de la configuration donnée. Dès lors, on considère la suite des différences entre les nombres de graines des cases de la configuration et du groupe de marche minorant maximal.

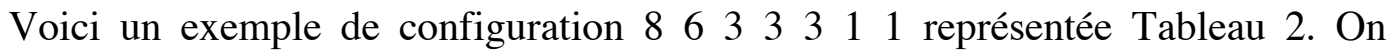
indique sur la ligne inférieure le groupe de marche minorant maximal (en gras) et deux lignes plus bas la suite des différences (en italiques). Le semis de la case de gauche contenant huit graines conduit à 74442211 , avec le même groupe de marche minorant maximal et une suite des différences modifiée :

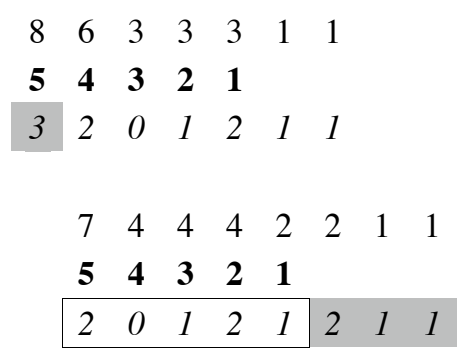

TABLEAU 2. Transformation de la suite des différences

lors du déplacement d'une configuration par semis de la case de gauche

Quel est l'effet de l'opération de déplacement sur la suite des différences? On voit que les trois graines de sa première case (grisées dans la position initiale) sont réparties à la fin (trois cases grisées dans la position d'arrivée), en laissant passer un nombre de cases exactement égal à la longueur du groupe de marche minorant maximal, c'est-à-dire cinq dans cet exemple (cases contenant 20012121$).$

Lorsque la configuration a le même nombre de cases que son groupe de marche minorant maximal, le déplacement fait apparaître un zéro supplémentaire dans la suite des différences, non présent dans la position initiale.

Un raisonnement utilisant le fait qu'une configuration périodique revient dans son état initial après plusieurs déplacements, dont on retracera les étapes ci-après, permet de montrer que, dans ce cas, la suite des différences ne peut contenir que des zéros ou des uns. Il en résulte que toute configuration périodique est nécessairement un groupe de marche augmenté. Pour donner une idée plus précise de l'argumentation, il faut examiner plus attentivement le déplacement des configurations résultant de semis 
successifs de leur case de gauche, et analyser les modifications induites sur la suite des différences.

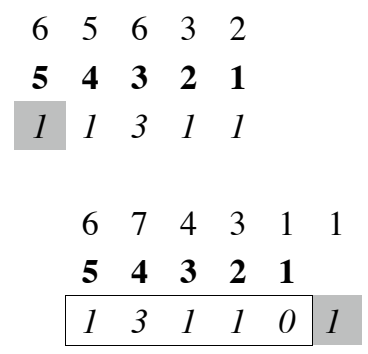

TABLEAU 3. Apparition d'un zéro dans la suite des différences lorsque la configuration a autant de cases que son groupe de marche minorant maximal

\subsubsection{Définitions formelles}

Formellement, on peut représenter une configuration de cases successives par un mot sur l'alphabet $\mathbb{N}$, ensemble des entiers naturels, c'est-à-dire une suite finie d'entiers indiquant le contenu de ces cases. En reprenant les conventions habituelles de la combinatoire des mots (cf [Lothaire, 1983]), on note $|w|$ la longueur d'un mot $w$, c'est-àdire le nombre de termes de la suite, et $w_{i}$ ses éléments pour $i$ compris entre 0 et $|w|-1$. La concaténation de deux mots $u$ et $v$ notée $u v$ est le mot obtenu en mettant les éléments de $v$ à la suite de $u$. On confond le mot de longueur un, ne comportant qu'un seul entier, avec cet entier lui-même. On appelle mot vide le mot de longueur nulle.

Si $w$ est un mot, on considère le groupe de marche $m$ le plus grand qui minore $w$, c'est-à-dire tel que $m_{i} \leq w_{i}$ pour tout $i<|m|$. On note $r$ la longueur de $m$ (avec $r \leq|w|$ ), et $d$ la suite des différences, c'est-à-dire le mot obtenu en retirant à chaque élément de $w$ l'élément correspondant de $m$.

Avec ces notations, l'opération $s(w)$ de déplacement des figures s'exprime comme une fonction de $d$, notée $s_{r}(d)$ avec $r=|m|$ :

$$
s(w)=m+s_{r}(d)
$$

dont la définition formelle est donnée plus loin. Précisons que la somme est effectuée ici sur des mots, c'est-à-dire qu'on additionne les éléments en même position, en recopiant éventuellement la fin du mot qui dépasse.

Le calcul de $s_{r}(d)$ s'effectue de la manière suivante. Différents cas se présentent selon que $|d|=r$ ou $|d|>r$ (rappelons qu'on ne peut avoir $|d|<r$ ). On note $d=x v$, où $x$ est le nombre contenu dans la première case de $d$, et $v$ le reste de la suite. Lorsque $|d|>$ $r$, on pose $v=u v^{\prime}$ avec $|u|=r$ ( $v^{\prime}$ éventuellement vide), et de nouveau, on distingue deux cas selon que $\left|v^{\prime}\right| \geq x$ ou non. Si c'est le cas, on pose $v^{\prime}=z z^{\prime}$ avec $|z|=x$ et $z^{\prime}$ le reste (notons que $z$ est vide si $x=0$ ). Si $\left|v^{\prime}\right|<x$, on définit $z$ en prolongeant $v^{\prime}$ avec des zéros de telle sorte que $|z|=x$ (et $z^{\prime}$ est le mot vide).

On peut alors donner une définition formelle de $s_{r}(d)$ :

(1) $\mathrm{Si}|d|=r$, on doit rajouter un zéro à la fin de $d$, ce qui s'écrit :

$$
s_{r}(d)=s_{r}(d 0)
$$


et qui ramène le calcul au cas suivant (2), car $|d 0|=r+1$.

(2) Si $|d|>r$, on répartit les $x$ graines de la première case de $d$ sur chaque case de $z$, en laissant passer exactement $|u|=r$ cases, ce qui s'écrit :

$$
s_{r}(d)=s_{r}\left(x u v^{\prime}\right)=s_{r}\left(x u z z^{\prime}\right)=u\left(z_{0}+1\right) \ldots\left(z_{x-1}+1\right) z^{\prime} .
$$

Dans la section suivante, on considère une configuration périodique $w$. On note $w^{j}$ la figure obtenue après $j$ déplacements, et par définition $w^{p}=w$ pour une certaine valeur $p$. En utilisant ces définitions, nous pouvons donner un aperçu de la preuve d'Henning Bruhn.

\subsection{3. Étapes de la preuve}

Henning Bruhn démontre que toute configuration périodique de l'awélé est nécessairement un groupe de marche augmenté au sens de Bouchet. Son raisonnement consiste à énoncer six propositions successives, donc chacune contient des observations assez simples sur les propriétés des configurations périodiques, mais dont l'enchaînement constitue un raisonnement complexe. Nous allons présenter chacune de ses étapes d'abord sous une forme intuitive qui ne nécessite aucun formalisme particulier, puis sous forme de démonstrations exprimées dans le formalisme décrit cidessus. On remarque d'abord que :

(1) Pour une configuration périodique, le « groupe de marche minorant maximal » est aussi le plus grand groupe de marche minorant de toutes les configurations obtenues en déplaçant la configuration initiale (par semis successifs de la case de gauche).

On voit facilement dans l'exemple du Tableau 3 ci-dessus, avec la configuration 6 5632 , que si la première case a plus de graines que celle de son groupe de marche minorant, le semis touche plus de cases que dans ce dernier. En effet, la case initiale contient six graines, alors que celle du groupe de marche minorant en contient cinq. Le semis augmente le contenu de six cases consécutives de la configuration initiale, ce qui donne 674311 , alors que dans le groupe de marche minorant, seules cinq cases voient leur contenu augmenter.

Dans le cas général, pour une configuration quelconque, périodique ou non, le nombre de cases touchées par le semis de la case de gauche, pour cette configuration, est toujours supérieur ou égal au nombre correspondant pour son groupe marche minorant maximal. On en déduit que la configuration obtenue après déplacement reste minorée par le même groupe de marche. En itérant le raisonnement, on voit que si un groupe de marche minore une configuration, périodique ou non, il minore également toutes les configurations obtenues par déplacements successifs. Mais quand la configuration est périodique, on revient à l'état initial après un certain nombre d'étapes. Il n'est donc pas possible qu'apparaisse parmi ces états successifs un groupe de marche minorant plus grand, car il serait aussi minorant de la configuration initiale. Donc pour une configuration périodique, le « groupe de marche minorant maximal » est aussi le groupe de marche minorant maximal de toutes les configurations obtenues par déplacements successifs.

On s'intéresse désormais à la suite des différences entre les configurations obtenues par déplacements de la configuration initiale et le groupe de marche minorant maximal. Notons $r$ le nombre de cases de ce dernier. Le raisonnement consiste à observer soigneusement l'évolution des $r+1$ premières valeurs de cette suite au cours 
des déplacements successifs. On va montrer que les nombres la constituant ne peuvent prendre comme valeurs que zéro ou un. Il en résultera que la configuration périodique est nécessairement un groupe de marche augmenté. On fait d'abord l'observation suivante :

(2) L'une des $r+1$ premières valeurs de la suite des différences est nulle ou non définie.

En effet, si toutes les cases de la configuration avaient strictement plus de graines que les cases correspondantes du groupe de marche minorant maximal, et si de surcroît la configuration avait une case supplémentaire à droite non nulle, alors on pourrait choisir un groupe de marche minorant plus grand, ce qui contredit l'hypothèse selon laquelle celui-ci est maximal. Une analyse plus fine conduit au constat suivant :

(3) Pour une configuration périodique, le nombre d'éléments non nuls parmi les $r+1$ premières valeurs de la suite des différences reste constant au cours des déplacements successifs.

On montre que ce nombre d'éléments non nuls ne peut que croître. Par exemple, dans les déplacements envisagés Tableaux 2 et 3 , on voit que tout élément non nul parmi les $r+1$ premières valeurs de la suite des différences, exceptée la première valeur, est conservé après déplacement sans être modifié. Quant à la première valeur, si elle est non nulle, le semis des graines en ajoute une en position $r+1$, donc un nouvel élément non nul apparaît.

Ces remarques s'appliquent au cas général, que la configuration soit périodique ou non : les $r+1$ premières valeurs de la suite des différences, exceptée la première valeur, sont toujours inchangées lors du déplacement par semis de la case de gauche, et une nouvelle valeur non nulle apparaît toujours en position $r+1$ lorsque la première valeur est non nulle. On voit en fin de compte que le nombre d'éléments non nuls parmi les $r+1$ premières valeurs de la suite des différences est croissant lors des déplacements. Lorsque la configuration est périodique et revient à son état initial, il en résulte que ce nombre ne peut être que constant.

(4) Pour une configuration périodique, si un élément supérieur ou égal à deux apparaît en tête de la suite des différences, il ne peut être suivi d'un zéro.

Cette situation correspond à la configuration représentée Tableau 4. La suite des différences 2011212 commence par un deux suivi d'un zéro. Si l'on effectue deux déplacements successifs, on obtient 1221211 . On constate que le nombre d'éléments non nuls parmi les $r+1$ premières valeurs ( $r$ vaut cinq) est passé de cinq à six. Or la proposition (3) montre que cette situation est impossible pour une configuration périodique, puisque ce nombre doit être constant.

Utilisons les définitions formelles ci-dessus pour démontrer la proposition (4). On, suppose que la suite des différences à l'étape $j \mathrm{~s}$ 'écrit $d^{j}=(2+x) 0 u z$, avec $x \geq 0$ et $\mathrm{I}(2+$ $x) 0 u l=r+1$. L'application de $s_{r}$ donne, en introduisant deux entiers $x^{\prime}, x^{\prime \prime} \geq 0$ :

$$
\begin{gathered}
s_{r}\left(d^{j}\right)=0 u\left(1+x^{\prime}\right)\left(1+x^{\prime \prime}\right) z^{\prime}, \quad \text { puis } \\
s_{r}^{2}\left(d^{j}\right)=u\left(1+x^{\prime}\right)\left(1+x^{\prime \prime}\right) z^{\prime}
\end{gathered}
$$

avec $\left|u\left(1+x^{\prime}\right)\left(1+x^{\prime \prime}\right)\right|=r+1$. On voit que $u\left(1+x^{\prime}\right)\left(1+x^{\prime \prime}\right)$ fait apparaître un élément non nul supplémentaire par rapport à $(2+x) 0 u$, ce qui contredit $(3)$, d'où la proposition (4). 


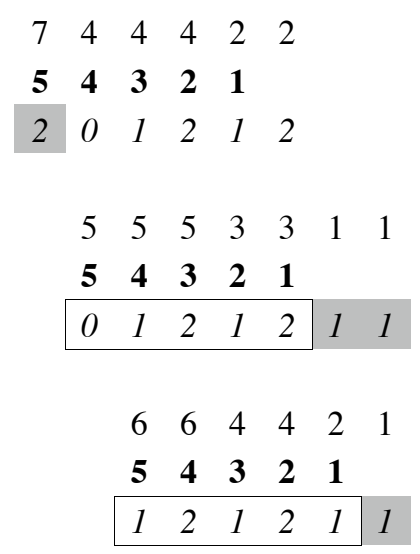

TABLEAU 4. La suite des différences comporte la succession 20. Le nombre d'éléments non nuls parmi les six premières valeurs n'est pas constant et passe de cinq à six

(5) Pour une configuration périodique, la suite des différences ne peut contenir que des zéros ou des uns.

L'idée, qui constitue le cœur de la démonstration, est que, si la suite des différences comporte une case supérieure ou égale à deux, suivie de cases valant un, puis d'une case nulle, alors le semis reproduit la même succession de valeurs après quelques étapes, mais où le nombre de cases intermédiaires (contenant un) décroît strictement d'une unité. Or une telle situation est impossible pour une figure périodique, car on doit revenir à l'état initial après plusieurs déplacements.

Cette situation est illustrée par le Tableau 5. La suite des différences vaut 21011 , le deux initial et le zéro étant séparés par un chiffre un. Après quatre déplacements, elle devient 1001201 , ce qui fait apparaître la succession 20 sans chiffre intermédiaire. En vertu de (4), la configuration initiale 75332 ne peut donc être périodique.

Pour démontrer formellement la proposition (5), supposons qu'il existe un élément de $d$ entre les positions 0 et $r$ contenant au moins deux graines. En effectuant des semis successifs, on peut placer cette case en tête de la configuration. D'après (2), il existe une case nulle entre les positions 0 et $r$. On cherche alors parmi les $d^{j}$ commençant avec une case supérieure ou égale à deux, celui dont le nombre $k$ d'éléments séparant cette case du premier zéro est minimal. Notons que d'après (4), on a $k \geq 1$, car l'élément supérieur ou égal à 2 ne peut être suivi de 0 . Le mot $d^{j}$ peut s'écrire :

$$
d^{j}=(2+x) 1 u 0 v
$$

avec $x \geq 0$ et $u$ un mot qui contient d'éventuels 1 supplémentaires, avec $|u|=k-1$. Conformément à la définition de $s_{r}$, on exprime le reste $v$ sous la forme $v=t z$, en complétant le cas échéant $t$ par des zéros de telle sorte que $|1 u 0 t|=r$ (et avec $z$ éventuellement vide). On obtient, en introduisant deux entiers $x^{\prime}, x^{\prime \prime} \geq 0$ :

$$
\begin{gathered}
s_{r}\left(d^{j}\right)=1 u 0 t\left(1+x^{\prime}\right)\left(1+x^{\prime \prime}\right) z^{\prime}, \\
s_{r}^{2}\left(d^{j}\right)=u 0 t\left(1+x^{\prime}\right)\left(2+x^{\prime \prime}\right) z^{\prime}, \\
\ldots \\
s_{r}^{k+2}\left(d^{j}\right)=0 t\left(1+x^{\prime}\right)\left(2+x^{\prime \prime}\right) u^{\prime} z^{\prime \prime},
\end{gathered}
$$




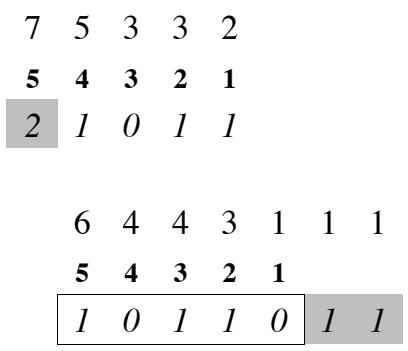

\begin{tabular}{llllll|}
5 & 5 & 4 & 2 & 2 & 2 \\
$\mathbf{5}$ & $\mathbf{4}$ & $\mathbf{3}$ & $\mathbf{2}$ & \multicolumn{1}{l}{$\mathbf{1}$} & \multicolumn{1}{l}{} \\
\cline { 1 - 4 } 0 & 1 & 1 & 0 & 1 & 2
\end{tabular}

$$
\begin{aligned}
& \begin{array}{lllll}
6 & 5 & 3 & 3 & 3
\end{array} \\
& \begin{array}{|lllll|}
\mathbf{5} & \mathbf{4} & \mathbf{3} & \mathbf{2} & \mathbf{1} \\
\hline 1 & 1 & 0 & 1 & 2 \\
\hline
\end{array} \\
& \begin{array}{llllll}
6 & 4 & 4 & 4 & 1 & 1
\end{array} \\
& \begin{array}{|lllll|l|}
\mathbf{5} & \mathbf{4} & \mathbf{3} & \mathbf{2} & \mathbf{1} & \\
\hline 1 & 0 & 1 & 2 & 0 & 1
\end{array}
\end{aligned}
$$

TABLEAU 5. La suite des différences comporte la succession 210. Après quatre déplacements, on voit apparaître la succession 20

avec $\left|u^{\prime}\right|=|u|$. En appliquant $s_{r}$ une fois de plus, on fait disparaître un zéro initial, donc si un élément apparaît en position $r$, il doit être nécessairement nul, car sinon, le nombre d'éléments non nuls augmenterait d'une unité, contrairement à (3) :

$$
s_{r}^{k+3}\left(d^{j}\right)=t\left(1+x^{\prime}\right)\left(2+x^{\prime \prime}\right) u^{\prime} 0 z^{\prime \prime \prime} .
$$

Mais il peut arriver aussi qu'aucun élément n'apparaisse en position $r$ (si $z$ " est vide). Or dans ce cas, un zéro réapparaît à l'étape suivante comme on le voit dans l'exemple du Tableau 5 ci-dessus (on note $y$ le premier élément de $t$, avec $t=y t^{\prime}$ ) :

$$
\begin{gathered}
s_{r}^{k+3}\left(d^{j}\right)=y t^{\prime}\left(1+x^{\prime}\right)\left(2+x^{\prime \prime}\right) u^{\prime}, \\
s_{r}^{k+4}\left(d^{j}\right)=t^{\prime}\left(1+x^{\prime}\right)\left(2+x^{\prime \prime}\right) u^{\prime} 0 y^{\prime} .
\end{gathered}
$$

Dans tous les cas, on voit se constituer la séquence $\left(2+x^{\prime \prime}\right) u^{\prime} 0$ qui commence par un élément supérieur ou égal à 2 , se termine par 0 , et dans laquelle la distance entre les deux est égale à $k-1$, donc strictement inférieure à $k$, ce qui est en contradiction avec la définition de $k$. Ceci démontre la proposition (5).

(6) Le nombre de cases d'une configuration périodique ne peut exéder de plus d'une unité celui de son groupe de marche minorant maximal, ce qui revient à dire que la longueur de la suite des différences est bornée supérieurement par $r+1$.

Dans le Tableau 5, la situation contraire est illustrée par la configuration 6443111 , qui a sept cases alors que son groupe de marche minorant maximal n'en a que cinq. Au cours du semis, l'une des graines de la case initiale de la suite des différences est mise dans une case non vide, ce qui fait apparaître une case ayant plus de 
deux graines. La configuration ne peut donc être périodique conformément à (5). Le cas où la case initiale de la suite des différences contient zéro graine est illustré par la configuration 5553311 dans le Tableau 4. Elle aussi comporte sept cases alors que son groupe de marche minorant maximal n'en a que cinq. Après déplacement, le zéro initial de la suite des différences disparaît alors qu'une case non vide apparaît à la fin, contredisant le fait que le nombre d'éléments non nuls doit rester constant d'après (3). Dans tous les cas, on voit qu'une configuration périodique ne peut comporter deux cases de plus que son groupe de marche minorant maximal.

La démonstration formelle de (6) consiste à supposer l'assertion fausse. On peut alors supposer l'existence d'un élément non nul $y \geq 1$ en position $(r+1)+k$ avec $k \geq 0$, et écrire :

$$
d=z u z^{\prime} y v
$$

en choisissant $z, u, z^{\prime}$ de telle sorte que $|z u|=r+1$ et $|z|=\left|z^{\prime}\right|=k$. En appliquant $k$ fois la fonction $s_{r}$, on a :

$$
s_{r}^{k}(d)=u z^{\prime \prime} y v .
$$

Soit $x$ le premier élément de $u$, et $u^{\prime}$ le reste, c'est-à-dire $u=x u^{\prime}$. Compte tenu de $x \leq 1$ d'après (5), et de $\left|u^{\prime} z "\right|=r$, il vient :

$$
s_{r}^{k+1}(d)=u^{\prime} z^{\prime \prime}(y+x) v .
$$

Si $x=1$, on a $y+x \geq 2$, ce qui contredit (5). Et si $x=0$, on perd un élément nul entre les positions 0 et $r$, pour le remplacer par un élément non nul $y$, ce qui contredit (3), d'où (6).

En conclusion, la figure périodique $w$ est nécessairement de longueur $|w| \leq r+1$ où $r$ est la longueur du plus grand groupe de marche minorant, et la suite des différences associée vérifie $d_{i} \leq 1$ pour tout $i$, ce qui montre que $w$ est un groupe de marche augmenté au sens de Bouchet.

\subsection{POSITIONS DÉTERMINISTES DANS LES FINS DE PARTIE}

\subsubsection{Définition des positions déterministes}

D'autres résultats mathématiques intéressants concernant l'awélé ont été obtenus en 1995 par D. M. Broline et D. E. Loeb. Ils se sont intéressés à des situations de fin de partie qu'ils appellent «positions déterministes » [Broline, Loeb, 1995] ${ }^{7}$. Il s'agit d'une position telle que :

(1) Sud peut capturer des graines à chaque coup,

(2) Nord n'a qu'une seule graine de son côté à chaque coup,

(3) toutes les graines, sauf une, sont finalement capturées par Sud.

Notons que le groupe de marche 21 à deux cases, lorsqu'il est placé à cheval entre Sud et Nord comme on l'a vu dans l'exemple de Bennett (Section 1.2.3), permet à Sud de prendre deux graines et constitue de ce fait une position déterministe, au sens de Broline et Loeb. Cette notion de position déterministe a des liens étroits avec ce que les joueurs africains appellent un «piège », comme on le verra plus loin.

\footnotetext{
${ }^{7}$ J'ai eu connaissance de ces travaux grâce aux conseils amicaux de Jean-Paul Allouche.
} 
Dans une position déterministe, la graine de Nord est nécessairement dans la case « a » si c'est à Nord de jouer, et la case « b » si c'est à Sud de jouer. En effet, quand Sud doit jouer, il faut qu'il effectue une prise en ne laissant qu'une seule graine dans le camp de Nord. Cela implique que la graine de Nord soit en position «b » et que Sud termine sa répartition dans cette case pour qu'il y ait capture. Sud prend alors le contenu de $\ll b$ » et laisse une graine en $« \mathrm{a} »$.

On voit donc que, pour être « jouable », c'est-à-dire respecter les conditions de la définition ci-dessus, la case jouée par Nord ou Sud doit être telle qu'après semis, on s'arrête dans la case « $\mathrm{b}$ ». Pour Nord, il s'agit de la case «a». Pour Sud, les cases possibles sont $« \mathrm{~F} »$ si elle contient deux graines, « $\mathrm{E} »$ si elle en contient trois, « $\mathrm{D} » \mathrm{si}$ elle en contient quatre, etc.

Broline et Loeb donnent l'exemple suivant :

0000010

000422

représenté Figure 9.

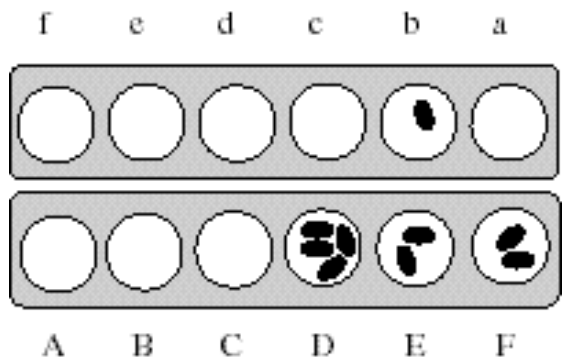

FIGURE 9. La «position déterministe » 42201 , la case jouée par Sud doit être « $\mathrm{F} »$

C'est à Sud de jouer, et la succession de coups « FaDaEaF » lui permet de prendre huit graines, et d'en laisser une dans le camp adverse en position « $\mathrm{a} »$.

Broline et Loeb font le raisonnement suivant, qui s'exprime naturellement sans recourir à un formalisme mathématique. Sud avait au départ deux cases «jouables» dans l'exemple précédent, à savoir « $\mathrm{F} »$ ou « $\mathrm{D} »$. Mais s'il avait joué « $\mathrm{D} »$, la case « F aurait reçu trois graines, entraînant la mise de plusieurs graines dans le camp adverse lors d'un coup ultérieur. On ne serait plus alors dans une position déterministe. Une conséquence de la définition des positions déterministes est que, parmi les cases « jouables », c'est-à-dire dont le nombre de graines leur permettent de terminer le semis dans la case $\langle\mathrm{b} »$, il faut jouer celle qui a le moins de graines, si on veut que le jeu reste dans une position déterministe.

Dans ce qui suit, l'expression « position déterministe à $s$ graines » désigne :

- lorsque $s$ est pair une position déterministe contenant $s+1$ graines en tout, et c'est à Nord de jouer ;

- lorsque $s$ est impair une position déterministe contenant $s$ graines en tout, et c'est à Sud de jouer.

La condition ci-dessus sur l'unicité du choix de la case à jouer parmi les cases « jouables » montre, de façon évidente, qu'il n'existe qu'une seule manière possible de 
passer d'une position déterministe à $s$ graines à une position déterministe à $s+1$ graines. Elle consiste à procéder de la manière suivante :

- on choisit la case vide la plus proche de la case « $b »$ en tournant dans le sens horaire, et on la remplit avec un nombre de graines égal à la distance qui la sépare de «b » (une pour « $\mathrm{a} »$, deux pour $« \mathrm{~F} »$, trois pour $« \mathrm{E} »$, etc.) de manière à en faire une case « jouable »;

- on enlève une graine à toutes les cases qui sont entre elle et « b ».

Par construction, cette nouvelle case sera la case «jouable» ayant le moins de graines, donc celle qui doit être jouée (par Nord si c'est «a », ou Sud dans les autres cas). Or en semant cette case, on reconstituera la position déterministe comportant $s$ graines dont on était parti. Il en résulte par induction que pour chaque valeur de $s, i l$ existe une seule position déterministe à s graines. Broline et Loeb en déduisent le tableau suivant. Les lignes impaires correspondent au cas où c'est à Nord de jouer, les lignes paires au cas où c'est à Sud (la graine de Nord est alors en position « $b$ », mais comme on l'a dit, elle n'est pas comptée dans la somme).

Prenons la dixième ligne de ce tableau. Elle contient la configuration 531101 , qui est la position déterministe à dix graines :

$$
\begin{array}{llllll}
0 & 0 & 0 & 0 & 1 & 0 \\
0 & 0 & 5 & 3 & 1 & 1 .
\end{array} \text {. }
$$

Sa seule case « jouable » est « $\mathrm{C} »$ du côté $\mathrm{Sud}$, unique case dont le semis conduit en « $\mathrm{b}$ » et permet à Sud de prendre deux graines, en laissant une graine en « $\mathrm{a} »$. On obtient alors la configuration 422 , qui est la position déterministe à neuf graines située une ligne au-dessus dans le tableau. Nord est alors obligé de jouer «a ». On passe ensuite à la position déterministe à huit graines 422 , qui est celle de l'exemple de Broline et Loeb cité plus haut (Figure 9). La seule case jouable devient « $\mathrm{F} »$ comme on l'a vu.

\begin{tabular}{|c|c|c|c|c|c|c|c|}
\hline Somme & A & B & C & D & E & F & a \\
\hline 1 & & & & & & & 1 \\
\hline 2 & & & & & & 2 & \\
\hline 3 & & & & & & 2 & 1 \\
\hline 4 & & & & & 3 & 1 & \\
\hline 5 & & & & & 3 & 1 & 1 \\
\hline 6 & & & & 4 & 2 & & \\
\hline 7 & & & & 4 & 2 & & 1 \\
\hline 8 & & & & 4 & 2 & 2 & \\
\hline 9 & & & & 4 & 2 & 2 & 1 \\
\hline 10 & & & 5 & 3 & 1 & 1 & \\
\hline 11 & & & 5 & 3 & 1 & 1 & 1 \\
\hline 12 & & 6 & 4 & 2 & & & \\
\hline 13 & & 6 & 4 & 2 & & & 1 \\
\hline
\end{tabular}

TABLEAU 6. Positions déterministes en fonction du nombre de graines

Broline et Loeb ont observé la courbe des valeurs du nombre minimal de graines possibles dans une position déterministe comportant $n$ cases. Par exemple, pour cinq 
cases (C D E F a), la plus petite position déterministe contient dix graines. Ils notent $s(n)$ ce nombre minimal de graines en fonction du nombre $n$ de cases, et les premières valeurs sont $s(1)=1, s(2)=2, s(3)=4, s(4)=6, s(5)=10, s(6)=12$.

Bien que cela n'ait pas de sens dans le cas de l'awélé, car le nombre de cases est fixe et égal à douze, Broline et Loeb se sont intéressés au comportement de $s(n)$ quand $n$ devient très grand. Ils obtiennent alors une formule décrivant ce comportement asymptotique :

$$
s(n) \sim n^{2} / \pi
$$

où l'on voit apparaître de façon inattendue, comme les mathématiques en ont le secret, le nombre $p i$.

\subsubsection{Apparition du nombre pi}

Sans entrer dans le détail de l'argumentation, qui recourt à des concepts mathématiques étrangers à tout contexte de tradition orale, indiquons seulement que l'apparition de $p i$ est liée à l'intervention de la fonction gamma d'Euler, et à sa valeur en 1/2 :

$$
\Gamma\left(\frac{1}{2}\right)=\sqrt{\pi}
$$

Les propriétés de cette fonction font que ses valeurs pour les demi-entiers se déduisent de la précédente en utilisant le produit des nombres impairs consécutifs :

$$
\Gamma\left(n+\frac{1}{2}\right)=\Gamma\left(\frac{1}{2}\right) \times\left(1-\frac{1}{2}\right)\left(2-\frac{1}{2}\right)\left(3-\frac{1}{2}\right) \ldots\left(n-\frac{1}{2}\right)=\sqrt{\pi} \times \frac{1 \times 3 \times 5 \ldots \times(2 n-1)}{2^{n}}
$$

La description complète du calcul de Broline et Loeb dépasserait le cadre de cet article, mais on peut indiquer seulement que, par un procédé usuel consistant à déployer une relation de récurrence du premier terme jusqu'à son terme courant, leur calcul fait apparaître le rapport du produit des nombres impairs consécutifs par celui des nombres pairs consécutifs. D'après ce qui précéde, ce rapport est relié à la fonction gamma et au nombre $p i$ :

$$
\frac{1 \times 3 \times 5 \ldots \times(2 n-1)}{2 \times 4 \times 6 \ldots \times 2 n}=\frac{1 \times 3 \times 5 \ldots \times(2 n-1)}{2^{n} \times n !}=\frac{1}{n ! \sqrt{\pi}} \times \Gamma\left(n+\frac{1}{2}\right)
$$

ce qui explique l'apparition de pi. La suite de la démonstration utilise une relation entre la fonction gamma et la fonction hypergéométrique de Gauss ${ }_{2} F_{1}$ qui permet de déterminer le comportement asymptotique cherché ${ }^{8}$.

\footnotetext{
${ }^{8}$ Les fonctions hypergéométriques sont les limites, lorsqu'elles convergent, des sommes de séries telles que le quotient du terme d'indice $k+1$ par le terme d'indice $k$ est une fonction rationnelle de $k$. La fonction qui intervient dans le calcul de Broline et Loeb s'écrit, avec la notation en usage pour ces fonctions :

$$
2 F 1\left(\frac{1}{2}, \frac{1}{2} ; 2 ; x\right)=\sum_{k=0}^{\infty} c_{k} x^{k}
$$

et elle est définie par $c_{0}=1$ et $\frac{c_{k+1}}{c_{k}}=\frac{(2 k+1)(2 k+1)}{(4 k+8)(k+1)}$.
} 


\section{ENQUÊTE DE TERRAIN SUR LE DISCOURS DES EXPERTS}

\subsection{LES TRAVAUX DE JEAN RETSCHITZKI}

\subsubsection{Enquête avec des experts de Côte d'Ivoire}

Les analyses précédentes portant sur certaines configurations particulières de l'awélé (groupes de marche augmentés, positions déterministes) sont-elles de pures questions mathématiques sans rapport avec la quête de stratégies gagnantes des joueurs? Notre propos est de montrer qu'il n'en est rien et que les experts du jeu raisonnent similairement. Le fait que les groupes de marche augmentés, par exemple, ne soient pas directement utiles à la conduite du jeu, et paraissent étrangers à toute préoccupation d'ordre stratégique, est sans importance pour notre propos. Il est clair que les experts africains ont des visées plus pratiques. Mais nous voulons montrer que leur approche rejoint celle des mathématiciens par plusieurs aspects : (a) mise en évidence de classes générales de configurations de graines ; (b) prédiction de l'évolution du jeu déterminée par ces configurations au moyen de raisonnements déductifs.

Nous allons pour cela analyser les explications données par des joueurs africains telles qu'elles sont décrites dans les recherches interculturelles menées par un spécialiste de psychologie cognitive, Jean Retschitzki, pour dégager ce qu'elles ont de commun avec les raisonnements mathématiques présentés dans les sections précédentes, et ce qui les en distingue le cas échéant. Ce chercheur s'est intéressé aux processus cognitifs mis en oeuvre par les joueurs d'awélé. Il développe tous les aspects du jeu : début de partie, milieu de partie comportant différentes stratégies de récolte ou de menace (avec tactiques défensives ou offensives) et fin de partie. L'un des concepts essentiels de la stratégie de l'awélé dans les milieux de partie est celle de "grenier» (appelé krou) : il s'agit d'une case dans laquelle on accumule des graines jusqu'au moment où le nombre de graines est suffisamment grand pour qu'en jouant cette case, on puisse effectuer des prises en terminant dans le camp de l'adversaire après avoir réalisé deux tours le long du tablier du jeu [Retschitzki, 1990, p. 46].

Une large place du livre de Jean Retschitzki est consacrée à la «théorie des fins de partie ». Compte tenu de notre approche comparative et du fait que les analyses mathématiques précédentes portaient essentiellement sur les fins de partie, nous allons nous limiter à cette phase du jeu. Retschitzki fait le constat suivant :

Les parties entre adultes sont très souvent interrompues bien avant que l'une
des règles relatives à la fin de jeu (un joueur n'a plus de graines; trop peu
de graines en jeu pour qu'une récolte soit encore possible) ne s'applique.
Ces interruptions par accord mutuel avec répartition des graines supposent
une bonne connaissance des cas possibles et/ou une anticipation à
relativement long terme [Retschitzki, 1990, p. 190].

Il a mené entre autres une enquête auprès de certains des meilleurs joueurs de la Côte d'Ivoire au début des années quatre-vingt, K. Wilson, A. Kouadio, K. Jean et Y. Kouadou. Le 3 avril 1983, il les interrogeait sur la manière de finir une partie.

\subsubsection{Notion de fin de partie canonique}

La réponse des experts ivoiriens concernant les fins de partie mettait en évidence la notion de situation « canonique »:

[Ils] nous ont exposé leur théorie des situations de fin de partie. Dans la plupart des cas, il s'agit de situations qu'on peut appeler 'canonique', les 
graines restantes étant distribuées dans les premières cases de la rangée de chaque joueur, à raison d'une graine par case [...] Nous reproduisons ciaprès quelques extraits significatifs de cette conversation pour illustrer le type de connaissances que possèdent les experts du jeu [Retschitzki 1990, p. 196].

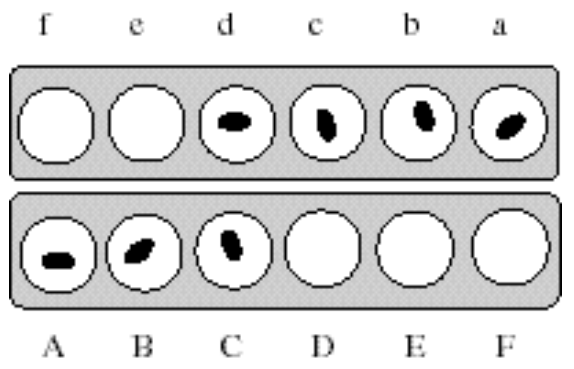

FIGURE 10. Une fin de partie « canonique » de type 3-4 analysée par des experts ivoiriens

On peut noter ces situations «canoniques » avec deux chiffres représentant le nombre de graines dans chaque camp, en plaçant en premier celui du joueur qui a la main. En voici une de type 3-4 analysée par les joueurs et représentée Figure 10 [Retschitzki, 1990, p. 197] :

0011111

111000.

Un expert ivoirien explique que si Sud commence, la situation conduit à un « piège » avec trait à Nord, dans lequel celui-ci prend nécessairement deux graines. Il montre à l'appui de sa démonstration une configuration, qualifiée par lui de "piège » pour Sud, en expliquant qu'on y est conduit inévitablement :

$$
\begin{array}{llllll}
1 & 3 & 0 & 0 & 0 & 0 \\
0 & 0 & 0 & 0 & 0 & 3 .
\end{array} \text {. }
$$

Comment fait-il ? Tout d'abord, pourquoi la situation « canonique » de départ (1) conduit-elle inévitablement à (2) ? Ensuite, pour quelles raisons Nord prend-il deux graines une fois arrivé dans cette situation (2) qualifiée de «piège »?

Analysons tout d'abord le passage de (1) à (2). Si Sud veut retarder le plus longtemps possible le moment où il doit placer des graines dans le camp de Nord, il arrive à la situation prédite par l'expert en $3+4+5=12$ coups. Il joue en effet d'abord « $\mathrm{CDE} »$, puis « $\mathrm{BCDE} »$, et enfin « $\mathrm{ABCDE} »$, soit douze coups. Mais dans ce cas, Nord effectue un parcours similaire en $2+2+3+4=11$ coups, en jouant les cases «de », puis «cd», puis « bcd » et enfin « abcd». À l'arrivée en (2), c'est donc à Nord de jouer. Tout autre déplacement de Sud comporterait moins de coups, l'obligeant à donner plus tôt des graines dans le camp de Nord et offrant à ce dernier plus de choix. Sud n'a donc guère la possibilité de s'écarter de la règle stratégique suivante: dans une fin de partie, éviter le plus possible de placer des pions dans le camp adverse. Si on ne la respecte pas, en effet, on augmente la mobilité de l'adversaire. En vertu de ce constat, Sud ne peut échapper à la situation (2).

\footnotetext{
${ }^{9}$ Le livre de Retschitzki rapporte également des analyses des situations de type 3-2, 3-3, 4-3, 5-4, 4-5, 3-4 et 5-5.
} 


\subsubsection{Notion de piège}

Il reste à voir pourquoi la situation (2) avec trait à Nord constitue un piège pour Sud. Cette notion de «piège » est analysée en détail par Retschitzski, qui la définit de la manière suivante :

La stratégie [des pièges] consiste à essayer de priver l'adversaire de graines, puis à lui en donner tout en créant une menace sur la ou les cases où il sera contraint de joue [Retschitzki, 1990, p. 50].

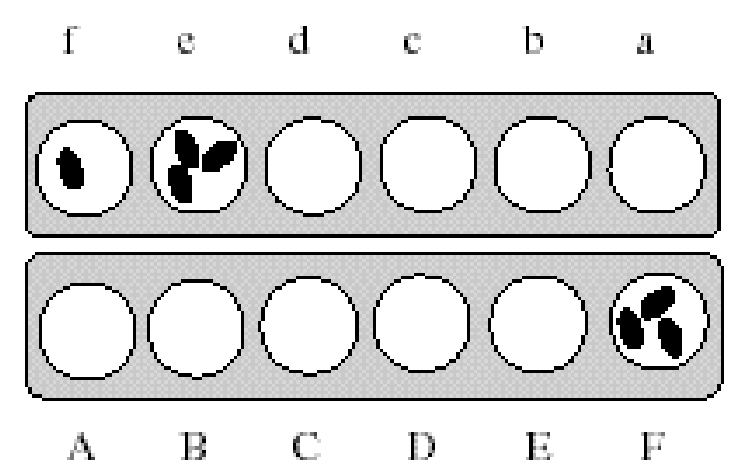

FIGURE 11. Une situation de « piège » où Nord prend deux graines

Plusieurs cas de piège sont possibles. La situation (2) représentée Figure 11 en est un. Le trait est à Nord, qui joue « $\mathrm{f} »$ :

\section{0 \\ 1000003.}

Sud a alors deux possibilités : ou bien il joue sa case de gauche «A graine est aussitôt prise par Nord; ou bien il joue sa case de droite « $\mathrm{F}$ » mais cela ne fait que reculer l'obstacle, car il donne des graines dans le camp de Nord qui va les jouer en attendant que Sud joue sa case de gauche. Dans tous les cas, l'expert ivoirien a raison : le piège conduit Nord à prendre deux graines. Sud doit jouer «A » (car jouer « $F »$ ne fait que retarder d'un coup l'obligation de jouer «A»), et Nord joue «e », ce qui lui permet de capturer deux graines en « B » et conduit à la situation (3) :

$$
\begin{array}{llllll}
10 & 0 & 0 & 0 & 0 \\
10 & 0 & 0 & 0 & 0 & 3 .
\end{array}
$$

Les travaux de Jean Retschitzski montrent que les joueurs d'awélé mettent en œuvre des processus cognitifs complexes, constitués pour une part de connaissances mémorisées en accumulant les expériences, mais aussi de raisonnements de type déductif appliqués à ces connaissances :

Ces joueurs adultes sont parfaitement capables de raisonnement de type hypothético-déductif. Ce résultat mérite d'être souligné car, même si cette capacité semble nécessaire pour être à même de résoudre les différents problèmes de la vie au niveau d'une communauté (interactions sociales, organisation des productions, etc.) peu d'études expérimentales dans des pays en voie de développement ont permis de mettre en évidence des raisonnements de type formel [Retschitzki, 1990, p. 199]. 


\subsection{RAISONNEMENT MATHÉMATIQUE ET DISCOURS DES EXPERTS}

\subsubsection{Existence de similitudes}

Il existe plusieurs similitudes entre les configurations analysées par l'expert ivoirien et celles que nous avons étudiées dans les sections précédentes. Tout d'abord, l'analyse permettant d'anticiper (2) à partir de la situation (1) ressemble aux "mouvements lents » définis par Bennett (Section 1.2.2).

Ensuite, la situation de «piège » (2) est très proche d'une position déterministe au sens de Broline et Loeb, à ceci près que Nord et Sud sont inversés, et que Sud a trois graines supplémentaires à droite (mais cela ne fait que retarder la logique d'enchaînement des coups comme on l'a vu). D'une manière plus générale, les positions déterministes se conforment précisément aux critères définissant les « pièges » au sens de Retschitzski. Elles en constituent une sorte de sous-classe particulière.

La position déterministe la plus proche de (2) (sans compter les trois graines à droite) aurait été celle correspondant à $s=4$ dans le Tableau 6 ci-dessus :

\section{0 \\ 0100003 .}

Nord aurait joué «e » (et non pas « $\mathrm{f} »$ comme dans la situation de l'expert ivoirien), ce qui aurait conduit au même gain, c'est-à-dire une capture de deux graines en $\ll B$ ».

La principale différence vient de ce que dans la situation (2) de l'expert ivoirien, l'analyse ne peut être réitérée à l'étape suivante (3). Autrement dit, elle n'est pas récursive. En effet, dans la situation (3) avec trait à Sud, celui-ci peut déplacer sa graine de gauche, et Nord ne pourra plus la capturer. La position déterministe correspondante aurait été (toujours sans compter les trois graines à droite) :

$$
\begin{array}{llllll}
2 & 0 & 0 & 0 & 0 & 0 \\
1 & 0 & 0 & 0 & 0 & 3
\end{array}
$$

où Sud aurait été obligé de jouer « $\mathrm{A} »($ soit immédiatement, soit après avoir joué « $\mathrm{F} »)$, puis Nord aurait effectué une deuxième capture, conformément aux enchaînements de coups analysés par Broline et Loeb dans les positions déterministes. Notons que cette situation (3bis) est aussi celle du groupe de marche à trois graines 21 analysé par Bennett (Section 1.2.3).

\subsubsection{Comparaison des raisonnements sur un plan général}

Il reste, pour conclure, à comparer sur un plan général les raisonnements des joueurs, tels qu'ils apparaissent dans les exemples étudiés par Retschitzski à ceux des mathématiciens présentés dans les sections précédentes. Chez ces derniers, le raisonnement comportait trois aspects :

a) repérer des classes générales de configurations,

b) prévoir par déduction l'évolution du jeu déterminée par ces configurations,

c) montrer que cette prévision est de portée générale, c'est-à-dire qu'elle est valable pour toutes les configurations d'une classe quelle que soit leur taille (ou, ce qui est équivalent, que le raisonnement ne dépend pas du nombre de graines des configurations). 
Par exemple, l'analyse de Bouchet-Bruhn met en évidence la classe des groupes de marche augmentés. Elle montre que les configurations de cette classe reviennent dans leur état initial après plusieurs semis de la case de gauche, autrement dit qu'elles évoluent de façon périodique. De plus, ce résultat est indépendant du nombre de graines de la configuration. Ce point mérite d'être souligné, car même si le tablier de l'awélé est borné, ce qui rend les configurations avec un grand nombre de graines sans intérêt dans la pratique, le fait de montrer que l'argumentation ne dépend pas du nombre de graines prouve qu'il s'agit d'une explication structurelle et non seulement d'un constat lié à un concours particulier de circonstances.

Chez les joueurs ivoiriens, les deux premiers aspects du raisonnement sont attestés dans les exemples que nous avons présentés plus haut :

a) L'existence de classes générales est illustrée de plusieurs manières, par les fins de partie canoniques ou les pièges. De plus, les premières définissent une classe potentiellement infinie (suites de uns consécutifs dans chaque camp), si l'on considère que les deux rangées du tablier ont un nombre illimité de cases.

b) La possibilité de prévoir l'évolution du jeu à partir d'éléments de ces classes apparaît également dans le discours des experts. Et il est important de noter qu'elle ne repose pas sur l'enchaînement de coups mémorisés, mais bien sur une argumentation procédant par déduction.

La distinction essentielle introduite en (b) est au cour des travaux de Jean Retschitzki :

La principale question à laquelle il s'agissait de répondre était de savoir si ces 'experts' villageois menaient une réflexion de type hypothético-déductive ou puisaient dans une sorte de répertoire des bons coups correspondant à une classe de situations [Retschitzki, 1990, p. 167].

Retschitzki donne plusieurs exemples de dialogues avec un expert autochtone qui font clairement apparaître que celui-ci procède de façon hypothético-déductive [Retschitzki, 1990, p. 181]. Au lieu d'enchaîner les coups mécaniquement, ce dernier procède par essais et erreurs, c'est-à-dire qu'il justifie le choix d'un coup en examinant les autres coups possibles et en raisonnant par élimination pour choisir le coup préférable. Le schème hypothético-déductif prend donc la forme de propositions conditionnelles : «si je joue cela, il se passe ceci ».

Le point qui semble distinguer l'approche des experts de celle des mathématiciens est le troisième (c). Pour autant qu'on puisse en juger, les experts ne formulent pas d'argumentation portant sur la structure des configurations indépendamment de leur taille. Il y a bien un effort pour mettre en évidence des caractères généraux, comme par exemple le fait qu'une fin de partie canonique conduise ou non à un piège. Et les experts tentent de classer les fins de partie selon ce critère. Mais ils procèdent plutôt par énumération de tous les cas que par un raisonnement applicable au cas général.

\section{CONCLUSION}

La question qui apparaît en filigrane de cette analyse est celle de l'universalité de la pensée rationnelle, sujet d'un débat ancien auquel Claude Lévi-Strauss avait apporté une contribution majeure au début des années 1960 avec son ouvrage devenu un classique de l'ethnologie, La Pensée sauvage. L'un des préjugés que ce livre contribuait à dissiper est celui du supposé manque d'efficacité pratique des connaissances développées dans 
les sociétés jadis qualifiées de «primitives». Des activités comme la magie ou la divination ont pu susciter chez les observateurs occidentaux une erreur d'appréciation les conduisant à considérer comme dénuées d'efficacité les techniques développées par ces sociétés. En réalité, à côté des techniques magiques ou divinatoires, celles-ci en développaient parallèlement d'autres à visées pratiques avec lesquelles elles parvenaient à une grande maîtrise de leur environnement en faisant preuve d'une démarche véritablement scientifique.

Mais la pensée dite «sauvage » ne s'exerce pas uniquement dans le champ des applications pratiques. C'est même l'une de ses caractéristiques mises en évidence par Lévi-Strauss de répondre principalement à des exigences intellectuelles, avant de satisfaire des besoins [Lévi-Strauss, 1962, p. 21]. Elle se manifeste dans de multiples domaines à visée non utilitaires, comme par exemple les arts ou les jeux. Dans ses différentes manifestations, elle témoigne des mêmes règles de fonctionnement que la pensée rationnelle occidentale, au point qu'on peut la qualifier, au même titre, de pensée logique. Il n'y a donc rien de paradoxal à s'interroger sur les possibilités d'observer dans les manifestations de la pensée sauvage certaines formes de mathématique, que l'ethnomathématique se donne comme objet d'étude et qu'elle s'efforce de découvrir dans des activités spécifiques telles que l'awélé.

$\mathrm{Au}$ terme des analyses que nous avons présentées, comment peut-on caractériser une « ethnomathématique de l'awélé » ? On voit qu'il est possible de mettre en relation, dans certains cas particuliers, le raisonnement des experts indigènes d'une part et celui de mathématiciens s'intéressant au jeu d'autre part. Nous avons restreint notre propos aux situations de fins de partie, dans la mesure où cet aspect du jeu apparaît de façon privilégiée dans les travaux réalisés par les mathématiciens sous prétexte de l'awélé, ce qui facilitait notre comparaison. Mais il est évident que cette réflexion demanderait à être complétée par une analyse des raisonnements utilisés par les joueurs dans le reste du déroulement des parties, par exemple la constitution de greniers (Section 3.1.1), et à tenter de les décrire dans un cadre mathématique.

Ainsi le prolongement naturel de cette approche, dans une perspective plus large, consisterait-il à formaliser les raisonnements des joueurs de la même manière que le font les mathématiciens. Cela reviendrait à donner une définition formelle des classes de configurations de graines sur lesquelles ils raisonnent (par exemple celles qu'on a étudiées précédemment: fins de partie canoniques, pièges, etc.), à expliciter l'articulation logique des déductions auxquelles ils procèdent à partir de ces configurations pour prévoir l'evolution du jeu, et le cas échéant, à évaluer dans quelle mesure ils sont conscients que leur argumentation repose sur des propriétés structurelles indépendantes de la taille des configurations envisagées. Grâce à ce type d'analyse, sur le thème de l'awélé comme sur d'autres sujets de même nature, on peut dépasser la simple description mathématique «hors contexte», et aborder la dimension véritablement « ethnomathématique » du problème. 


\section{BIBLIOGRAPHIE}

ASCHER M., ASCHER R., "Ethnomathematics”, History of science, XXIV, 1986, p. 125-144.

BENETT G.T., "Wari”, Religion \& art in Ashanti, R.S. Rattray (ed.), Oxford, The Clarendon Press, 1927.

BERGE C., Théorie générale des jeux à n personnes, Mémoire des sciences mathématiques t. 138, Paris, Gauthier-Villars, 1957.

BOUCHET A., "Owari I. Marching groups and periodical queues”, manuscrit, 2005.

http://www.rpi.edu/ eglash/isgem.dir/texts.dir/OwariI.pdf

BOUCHET A., “Owari II. Marching groups and bulgarian solitaire”, manuscrit, 2007.

http://www.rpi.edu/ eglash/isgem.dir/texts.dir/OwariII.pdf

BOYER P., « Tradition et vérité », L'Homme, XXIV, 1986, p. 309-329.

BROLINE D. M., LOEB D.E., "The combinatorics of mancala-type games : Ayo, Tchoukaillon, and $1 / \pi$ ", UMAP Journal (The Journal of Undergraduate Mathematics and its Applications) 16 (1), 1995, p. 21-36.

BRUHN H., "Periodical states and marching groups in a closed owari", Discrete Mathematics, 2008 [à paraître].

CAILlOIS R. (éd.), « Jeux et sports », Encyclopédie de la Pléiade, 1967.

CHEMILLIER M., «Mathématiques et musiques de tradition orale», Rencontres musicales pluridisciplinaires, Lyon, mars 1996, H. Genevois, Y. Orlarey (éds.), Musique \& mathématiques, Lyon, Aléas-Grame, 1997, p. 133-143.

CHEMILLIER M., Les mathématiques naturelles, Paris, Odile Jacob, 2007(a).

CHEMILLIER M., «Mathématiques de tradition orale », Mathématiques et Sciences humaines 178, 2007(b), p. 11-40.

CONWAY J. H., On numbers and games, Academic Press, 1976.

DAMPIERRE É (de), « Le jeu nzakara de la guerre », note MSHO n 1, Université Paris X.

DAVIS P. J., HERSH R., L'univers mathématique, [trad. Lucien Chambadal], Paris, Gauthier-Villars, 1985. DELEDICQ A., POPOVA A., Wari et solo. Le jeu de calculs africain, Paris, CEDIC, 1977.

EGLASH R., « L'algorithmique ethnique », Pour la science, dossier spécial, n 47, 2005, p. 102-104.

HUIZINGA J., Homo ludens : essai sur la fonction sociale du jeu, 1938, [réédition Paris, Gallimard, collection Tel, 1988].

KOVACH R. P., Oware!, A winning numbers game, Sapient Software, 1995, [svn.net/rkovach/oware (contient en annexe la reproduction du chapitre de G.T. Bennett)].

LÉVI-STRAUSS C., La pensée sauvage, Paris, Plon, 1962, [rééd. Agora].

LOTHAIRE M., Combinatorics on words, Encyclopedia of Mathematics, Vol. 17, Addison-Wesley, 1983, [reprinted Cambridge University Press, 1997].

RATTRAY R. S. (Capt.) (éd.), Religion \& art in Ashanti, Oxford, The Clarendon Press, 1927, [le chapitre «Wari » est rédigé par G.T. Bennett].

RETSCHITZKI J., Stratégies des joueurs d'awélé, Paris, L’Harmattan, 1990.

ROMEIN J. W., BAL H.E., "Solving the game of Awari with parallel retrograde analysis", IEEE Computer 36 (10), 2003, p. 26-33.

SCRIBNER S., "Modes of thinking and ways of speaking. Culture and logic reconsidered", Thinking, readings in cognitive science, P. N. Johnson-Laird, P. C. Watson (eds.), Cambridge, Cambridge University Press, 1977, p. 483-500.

WOLFRAM S., “Cellular automata as models of complexity”, Nature t. 311, 1984, p. 419-424. 